Artículo Original

Recibido para publicación: Octubre 2 de 2016.

Aceptado para publicación: Noviembre 18 de 2016

\title{
Principio de transparencia en la contratación pública en el distrito de Cartagena, D. T. Y C.
}

\section{Principle of transparency in public procurement in the district of Cartagena D. T. y C.}

\author{
Autores: Milton Buelvas Mendoza ${ }^{1}$ \\ Estudiantes asistentes: \\ Martha Bermúdez Castellar, Isela Pérez Jiménez, Angie Sierra Vanegas² \\ Correspondencia: milton.buelvas@curnvirtual.edu.co - myenbume@gmail.com.
}

RESUMEN

El presente artículo de reflexión tiene como propósito analizar las contradicciones y desajustes al sistema de Contratación Estatal colombiano, en el marco de nuestro Sistema y Régimen Políticos, abordando un análisis que permita entender el tema de Gobernabilidad, Democracia, instituciones, normativas, sociedad, cultura política, participación, cuando de enfrentar la corrupción se trate. Asimismo, se abordara la experiencia que tuvo el sector descentralizado de Cartagena D. T. y C., durante el periodo 2008 al 2011,como forma de conocimiento de la inspección, vigilancia, visión ciudadana y control de la gestión en el periodo mencionado, Las conclusiones a las que llega el presente documento, pueden ser punto de partida para entender mucho mejor la problemática, y encontrar posibles soluciones: Instituciones fuertes, gobernabilidad, normativas, participación proactiva y consciente de la sociedad civil.

\section{Palabras Claves}

Gobernabilidad, Democracia, Contratación Administrativa, Control Social, Estado, Formación Ciudadana

\begin{abstract}
This reflection article aims to analyze the contradictions and imbalances Colombian State Procurement system as part of our System and Political System, addressing an analysis to understand the issue of governance, democracy, institutions, policies, society, culture political participation, when tackling corruption case. Also, his experience decentralized sector and C. DT Cartagena during the period 2008 to 2011, as a form of knowledge of the inspection, surveillance, public vision and management control in the aforementioned period will address The conclusions which arrives herein, may be starting to understand better the problems and possible solutions: strong institutions, governance, regulations, proactive and conscious participation of the civil society.
\end{abstract}

\section{Keywords}

Governance, Democracy, Public Procurement, Social Control, State, Citizenship Education.

\footnotetext{
${ }^{1}$ Abogado. Especialista en Seguridad Social y en Derecho Administrativo. Líder del Grupo Derecho Público de la facultad de ciencias sociales y humanas - Corporación Universitaria Rafael Núñez. Línea de investigación DESC - Eje de Gobernabilidad Democrática.

${ }^{2}$ Estudiantes del programa de derecho de la Corporación Universitaria Rafael Núñez, sede Cartagena, Investigadores Auxiliar del grupo de Derecho Público de la facultad de ciencias sociales y humanas. Línea de investigación DESC - Eje de Gobernabilidad Democrática. e-mail: asierrav13@curn.edu.co
} 


\section{Introducción.}

Desde los tiempos más remotos, el hombre por ser un ser social por naturaleza, ha tenido la gran necesidad de agruparse y con ello formar sociedades, y a partir de esas sociedades formar las diferentes relaciones con los demás países como se conocen hoy día. Movido por esa necesidad, surge la definición de lo que hoy se conoce como Estado, derivado de unos elementos esenciales e indispensables que lo integran como lo es territorio, la población, la soberanía, el poder público, y por último el reconocimiento internacional, todo esto, enmarcado dentro de las necesidades de sus habitantes, del ordenamiento jurídico y de la respetiva normatividad que lo integra y lo rige, como la Constitución-norma suprema y demás leyes y tratados internacionales introducidos a nuestra carta magna a través del bloque de constitucionalidad. Tal como se hace mención de la autonomía y soberanía que tiene nuestro Estado colombiano, consagrado en su preámbulo y en sus primeros artículos como principios, de igual forma también sucede con los demás Estados. Pero el nuestro parte de un poder que se ha sido entregado por el pueblo, sus habitantes, con la debida facultad para dirigir y gobernar. Por ello, se deben de cumplir con un mínimo de garantías que bien lo expresa la constitución en su artículo 2 como fines del Estado.

De tal forma, que son las autoridad las encargas y responsables de promover no solo el orden social sino también el bienestar de cada uno de sus habitantes y es de ahí donde surge y parte el tema a debatir en la presente investigación. Por lo que se nos hace necesario preguntarnos ¿cuál es el tratamiento que nuestros funcionarios y encargados de administrar justicia le dan a los recursos de todos sus ciudadanos? ¿Si efectivamente se cumplen a cabalidad todos los procedimientos que la ley estima para la contracción estatal?. Luego entonces, el 
principio de la contratación pública debe ser abordada desde una visión, que analice el fenómeno desde la misma concepción de lo que se debe entender por Estado y sus Sistemas y Regímenes Políticos. Lo anterior nos lleva a emprender uno de los enfoques metodológicos más interesantes para abordar un intento de explicar las contradicciones y desajustes de nuestro sistema de Contratación Estatal, en el marco de nuestro Sistema y Régimen Políticos, abordando un análisis dentro de un marco de Gobernabilidad y Democracia.

Teniendo en cuenta lo antes dicho, el Objetivo general que plantea esta investigación: es si ¿Han sido eficaces y efectivos los controles aplicados a los procesos de contratación estatal en el sector descentralizado de Cartagena D.T. y C., durante el período 2008-2011?, y como específicos Determinar Especificar la manera en que la gobernabilidad se soporte en buenas prácticas de contratación estatal y no se base en fenómenos de corrupción. Incentivar la intervención de la comunidad en cuanto a su aporte en el control que deben ejercer en concordancia con los principios y procesos de contratación estatal y fomentar en materia de contratación estatal una cultura de valores entre los funcionarios públicos que ejercen control posterior y sancionan las conductas corruptas en materia de contratación estatal.

\section{Método.}

La metodología empleada en esta investigación fue de carácter Socio jurídica, orientada bajo el enfoque de Tipo Cualitativo, y de tipo exploratorio, aproximándose a estructuras teóricas y fenomenológicas, teniendo como área de trabajo el distrito de Cartagena. Además por su carácter bibliográfico, lo que responde a la recolección de información, con base en textos de actualidad 
jurídico administrativo y el análisis sistemático, bajo parámetros de exploración rigurosa, es una investigación también de tipo jurídico.

\section{Materiales e Instrumentos De Recolección De La Información.}

Dado que el diseño metodológico es de carácter bibliográfico, se pretende recurrir en consecuencia a las fuentes secundarias que nutran y den contenido a la presente investigación, indagando en textos jurídicos, históricos, epistemológicos y filosóficos, buscando la información necesaria y pertinente que ayude a la solución del presente problema de investigación.

\section{Plan De Análisis De Los Datos.}

La comprensión, el análisis y la producción de textos jurídicos y filosóficos, se hará bajo parámetros de interpretación sistemática, lógico, racional de los textos.

\section{Unidad de Análisis.}

Esta Investigación, en la medida en que es exploratoria, trabajará con las siguientes unidades de análisis:

Actores Políticos: Los actores políticos y administración del sector descentralizado de Cartagena D.T. y C.

Actores Académicos: La Academia es un actor que históricamente ha sido muy activa en los procesos o movimientos de fomento de la transparencia y en contra de las conductas indebidas o corruptas en Colombia. No obstante, para efectos de esta investigación solo se tomará como Unidades de Análisis una muestra de expertos sacados de las Universidades y centros de pensamiento e investigación ubicados en la ciudad de Cartagena. 
Sociedad Civil: La sociedad y los líderes de los grupos sociales juegan un papel importante en cuanto a los procesos de vigilancia, control y sanción social de las conductas indebidas en materia de contratación estatal. Para esta investigación es importante consultar la visión, la perspectiva de sectores representativos de la Sociedad Civil del distrito de Cartagena, mediante la aplicación de Entrevistas Estructuradas, de tal manera que se pueda realizar un posterior análisis de la información

\section{Resultados Y Discusión}

Para el periodo comprendido en nuestra investigación año 2008 - 2011 se obtuvieron resultados inesperados en cuanto a los procesos de contratación que superaron lo imprevisto, para un total de 18 proponentes, cuando el número máximo era de dos y tres proponentes para cada proceso a partir de la entrada en vigencia del Plan de Desarrollo, denominado "Por una sola Cartagena", de igual forma se logró que a través de la Junta Administradora Local en el Plan Local de Desarrollo de cada localidad se recuperara la confianza de los ciudadanos en las instituciones y en la acción colectiva con el fin de proponer el crecimiento económico sostenido con competitividad, por construir una ciudad sostenible a través de estrategia financiera de los proyectos de emprendimiento, educación y culturización por tener una ciudad sostenible soñada, por lo que diseñaron políticas de fortalecimiento al sistema desconcentrado de localidades, dándoles facultades a los alcaldes locales para contratar las obras y servicios definidas por la Junta Administradora Local en el Plan Local de Desarrollo de cada localidad.

Lo anterior en complemento con normas, como el decreto 0928 del 4 de diciembre de 2008, sobre el programa de optimización de procesos por medio del cual se adoptó el sistema de gestión de calidad y el modelo estándar del control interno para la Alcaldía de Cartagena. 
De igual forma, se conoció que para la administración de ese año, el 4 de diciembre de 2008 se aprobó el Código de Ética de la Alcaldía Mayor de Cartagena de Indias, donde se establecieron los valores en general, los valores para todos los servidores públicos, los principios éticos y los valores éticos del servidor público, orientaciones del Código de Ética, roles y objetivos del servidor público, entre otras disposiciones.

Que busca este código de la ética: que el “... que esté vinculado o se vincule en un futuro a la Alcaldía Distrital de Cartagena, con una nueva cultura y renovado sentido de la labor que adelantamos en favor del bienestar de la ciudadanía. Esta herramienta guía la cultura organizacional del distrito hacia ambientes de cooperación, solidaridad, colaboración y transparencia en el manejo de los recursos públicos. Igualmente orienta la gestión de las servidoras y los servidores públicos en todos los niveles de la Alcaldía Distrital de Cartagena frente a los principios, valores y directrices institucionales, que regulan las relaciones laborales entre las servidoras y los servidores públicos y de éstos con los usuarios de los servicios que presta, para el logro de la misión, visión y el desarrollo integral de la ciudad a través del proceso administrativo, en aras de garantizar el bienestar de las cartageneras y los cartageneros, dentro de la transformación y exigencias de la globalización mundial" (Código de Ética, 2008).

Por otro lado también nos encontramos con el código del Buen Gobierno el cual tiene como propósito lograr una administración eficaz, eficiente, transparente lejos de todo acto de corrupción. Por lo que de una u otra manera estos proyectos y programas, han ayudado significativamente a que se dé el buen funcionamiento de la administración pública en cuanto al impulso e incentivo de una nueva cultura del funcionario, en el que desde el período de gobierno 2008-2011, ha contribuido de gran manera a una mejoría palpable en el terreno de la ética pública. 
Para el 2009 se aplicaron normas de nivel Nacional como la Ley 594 de 2009, en lo concerniente al archivo distrital la Alcaldía de Cartagena implantó oficialmente el Consejo de Archivo, iniciando labores propias de su función, citando a las reuniones reglamentarias, al ponerse en funcionamiento el Comité de Archivo Distrital, mediante el Decreto 4821 del 26 de septiembre del 2011. Ya para el año 2010 se proyecta la elaboración de la asistencia técnica para los procesos de planificación y administración municipal, en el acompañamiento a pactos de integridad y procesos de empalme entre gobiernos entrantes y salientes, así como de rendición de cuentas, para contribuir a un mejoramiento de la transparencia y eficacia de la administración pública; esto a su turno contribuirá a lograr una mejor asignación de los recursos públicos y la participación ciudadana.

Así mismo para el año del 2011, la Contraloría Distrital adopta el informe consolidado de auditoría gubernamental con enfoque integral; el documento compendia los resultados obtenidos en la evaluación de la totalidad de los puntos de control por medio de los cuales se ejerce el control a la Alcaldía Mayor y con ello, la expedición del Acuerdo 004 del 4 de mayo del 2011, por medio del cual se institucionaliza el sistema de gobernabilidad SIGOB para el distrito de Cartagena, cuyo objeto principal, como SIGOB, ha sido el desarrollo de enfoques, métodos, procesos de trabajo y herramientas informáticas para el soporte de la acción política gubernamental de alta dirección, contribuyendo con estos ámbitos institucionales que por sus competencias tiene un impacto directo en las condiciones de gobernabilidad necesaria para el desarrollo.

\section{Conclusiones}

Para avanzar en la lucha contra la corrupción se necesita más educación y culturización en cuanto a la escasa participación ciudadana y la baja formación 
ciudadana contribuye a que no exista control social sobre la contratación estatal, La distribución funcional del Estado colombiano en su sector descentralizado posibilita mayor eficacia y eficiencia en la contratación estatal, El sistema de controles internos y externos con que cuenta el Estado colombiano, aplicado al sector descentralizado del Distrito de Cartagena D. T. y C., reducirían los riesgos de ocurrencia de irregularidades y las prácticas de corrupción percibidas por el ciudadano en los procesos de contratación administrativa en sus diversas entidades.

La cooperación internacional y contar con la información adecuada y oportuna, que, por ejemplo, los sancionados por responsabilidad fiscal aparezcan en las centrales de riesgo con todo lo que ella significa. En la medida en que el ámbito local creció y tuvo mayores responsabilidades y recursos fue más riesgoso caer en actos de corrupción, pues la debilidad institucional local fue palpable, conjugada con el mayor poder de los políticos locales y el rezago evidente de los órganos de control. Las malas prácticas en la gestión estatal en cualesquiera de sus instancias, los actos de corrupción traen consigo el saboteo al cumplimiento de las labores misionales del Estado Social de Derecho, “...en la medida en que concentran en manos de unos pocos los recursos y bienes que deberían destinarse a intereses colectivos; distorsionan la toma de decisiones a favor de intereses particulares; aumentan los costos de administración de bienes y servicios públicos y privados; debilitan el respeto por la autoridad, erosionan la confianza ciudadana en las instituciones, y contribuyen a debilitar la legitimidad del Estado" (Ungar, 2010).

La participación activa, voluntaria, con criterios éticos, autónoma, en el sentido en que nadie sea obligado a participar. Es una decisión individual. Pero no existe una relación directa entre la eficacia de esa participación y el número de participantes. Cuando la participación es numerosa, masiva, el peso, el significado de cada individuo mengua, es una simple operación de numerador entre denominador 
(Sartori, 2008). Y entonces surgen, o pueden surgir, 'iluminados' que se ponen al frente de la democracia participativa, que pueden desdibujarla, apropiándose de la democracia, del Estado, para su propio beneficio, pero en forma participativa.

\section{Referencias.}

Agenda Ciudadana Por La Transparencia- Bolivar Y Cartagena (2011)http://www.funcicar.org/sites/default/files/archivos/agenda bolivar impres.pdf [consulta: sábado 18 de abril de 2015]

Bayuelo Schoonewolff, P. (2015). La educación y el Derecho en torno a un nuevo paradigma transformador. Justicia, 20(27). DOI: https://doi.org/10.17081/just.20.27.812

Benavides Vanegas, F. (2015). Soberanía, globalización y la constitucion de significado. Justicia, 20(27). https://doi.org/10.17081/just.20.27.811

Clavijo Cáceres, D. (2015). El enfoque de competencias en la formación del abogado para el siglo XXI. Justicia, 20(27). https://doi.org/10.17081/just.20.27.813

Constitución Política De Colombia. Legis Editores S.A. Bogotá. 1aㅡ edición. 1996.

González Geraldino, F. (2014). Sobre la memoria. Justicia, 19(26). https://doi.org/10.17081/just.19.26.828 
Hernández Sampieri, Roberto; Fernández-Colado, Carlos; Baptista Lucio, Pilar. Metodología de la Investigación. Cuarta Edición. McGraw Hill / Interamericana Editores, S.A. DE C.V. México, 2006.

Keefer, Snack, 2002, en el informe "El Conflicto, callejón con salida" - Informe Nacional de Desarrollo Humano Colombia 2003 - de las Naciones Unidas.

Periódico El Espectador, Destituido e inhabilitado gobernador de Bolívar Joaco Berrio (2014). http://www.elespectador.com/articulo190041-destituido-einhabilitado-gobernador-de-bolivar-joaco-berrio [consulta: viernes,28 de marzo de 2014]

Petro González, I. (2016). La sociologización del pensamiento jurídico. Justicia, 21(29). https://doi.org/10.17081/just.21.29.1232

Programa de las Naciones Unidas para el Desarrollo -PNUD- Colombia (2011). Evaluación externa de los resultados del proyecto 'Transparencia en la gestión pública a nivel territorial en Colombia'.

Programa de las Naciones Unidas para el Desarrollo -PNUD- Colombia (diciembre 2011). Buenas prácticas de transparencia en la gestión contractual.

http://www.pnud.org.co/img_upload/33323133323161646164616461646 164/gobierno/Libro\%20buenas\%20practicas.pdf[ Consulta: viernes 17 de abril de 2015]

Sánchez Novoa, P. (2015). Hacia un proceso monitorio en Colombia. Justicia, 20(28). https://doi.org/10.17081/just.20.28.1045 
Transparencia por Colombia. Capitulo Transparencia Internacional. La corrupción en Colombia - radiografía y retos. :http://www.transparenciacolombia.org.co/index.php?option=com_conten t\&view=article\&id=107\&Itemid=536[ Consulta: sábado 18 de abril de 2015]

Transparencia por Colombia. Capitulo Transparencia Internacional. La corrupción en Colombia - radiografía y retos. http://www.transparenciacolombia.org.co/index.php?option=com_content \&view=article\&id=107\&ltemid=536[ Consulta: domingo 19 de abril de 2015] 